\title{
Integration of Copper-Based and Reduced-Risk Fungicides for Control of Blumeriella jaapii on Sour Cherry
}

Patricia S. McManus, Department of Plant Pathology, University of Wisconsin, Madison 53706; Tyre J. Proffer, Department of Plant Pathology, Michigan State University, East Lansing 48824 and Department of Biological Sciences, Kent State University, Salem, OH 44460; Raffaele Berardi, Department of Plant Pathology, Michigan State University, East Lansing 48824 and Dipartimento di Protezione e Valorizzazione Agroalimentare, University of Bologna, Bologna, Italy; Barrett R. Gruber, Department of Plant Pathology, University of Wisconsin, Madison 53706; James E. Nugent, Northwest Michigan Horticultural Research Station, Traverse City 49684; and Gail R. Ehret, Zhonghua Ma, and George W. Sundin, Department of Plant Pathology, Michigan State University, East Lansing 48824

\begin{abstract}
McManus, P. S., Proffer, T. J., Berardi, R., Gruber, B. R., Nugent, J. E., Ehret, G. R., Ma, Z., and Sundin, G. W. 2007. Integration of copper-based and reduced-risk fungicides for control of Blumeriella jaapii on sour cherry. Plant Dis. 91:294-300.

Practical resistance to sterol demethylation inhibitor (DMI) fungicides among populations of Blumeriella jaapii, the cherry leaf spot (CLS) pathogen, was documented in 2005. In the present study, strategies to reduce selection for DMI-resistant strains of B. jaapii and adapt to possible restrictions on the use of chlorothalonil are described. Ten field trials were conducted on the sour cherry cultivars Balaton and Montmorency to test the efficacy of integrating respiration-inhibitor and copper-based fungicides into spray programs. Programs that included up to three sprays of copper-based fungicides were among the most effective for controlling CLS, although leaf phytotoxicity was sometimes observed. Under high disease pressure, eliminating chlorothalonil compromised CLS control. 'Balaton' and 'Montmorency' did not differ in the percentage of leaves with CLS or defoliation resulting from CLS. The physical modes of action of representative DMI, QoI, and copper-based fungicides were evaluated in a leaf disk assay. Trifloxystrobin, a QoI fungicide, provided the best protection against infection by $B$. jaapii. All fungicides were more effective than water when applied $46 \mathrm{~h}$ postinfection, although differences were not statistically significant in one of two trials. Tebuconazole, a DMI, was the only fungicide that was more effective than water in preventing resporulation from existing lesions in both trials. Isolates of B. jaapii, which varied in DMI-sensitivity, all were sensitive to copper in vitro.
\end{abstract}

Cherry leaf spot (CLS), caused by the fungus Blumeriella jaapii (Rehm) Arx, significantly reduces profits for sour cherry growers in the Great Lakes region of the United States every year. Left unmanaged, CLS causes defoliation by midsummer, which results in soft, poorly colored fruit that are low in soluble solids. Early defoliation delays acclimation of fruit buds and wood to cold temperatures in the fall, increases tree mortality during severe winters, and reduces fruit bud survival and fruit set the following year (7).

A combination of biological, economic, and regulatory factors puts the U.S. sour cherry industry in a precarious position regarding disease management. First, the cultivar Montmorency, which comprises nearly $100 \%$ of sour cherry production in the United States, is highly susceptible to

Corresponding author: P. S. McManus

E-mail: psm@plantpath.wisc.edu

Accepted for publication 3 October 2006.

DOI: 10.1094/PDIS-91-3-0294

(C) 2007 The American Phytopathological Society
CLS. Sources of resistance have been identified $(1,21,25)$, but resistance has not been incorporated into cultivars that meet the needs of producers in North America. Managing CLS in the Great Lakes region typically requires five to seven fungicide applications per year, starting at about the time of petal fall and continuing until late summer. The most important fungicides are chlorothalonil, captan, and several sterol demethylation inhibitor (DMI) fungicides including fenarimol, fenbuconazole, myclobutanil, and tebuconazole. Because of residue concerns, chlorothalonil may not be applied after the phenological stage known as "shuck split," which occurs about 1 week after petal fall, although it may be used again after harvest. Although chlorothalonil and captan, both classified by the U.S. Environmental Protection Agency (EPA) as B2 carcinogens, survived reassessment since implementation of the Food Quality Protection Act (FQPA), their use on major crops in the future may force registrants to cut uses on relatively minor crops, such as sour cherry, in order to retain more profitable uses.

Because DMI fungicides may be used season long, are generally less expensive than chlorothalonil, and are active against brown rot and powdery mildew as well as CLS $(11,12,16,18,22)$, they have been used intensively, and sometimes exclusively, in season-long disease control programs. However, intensive use of the DMI fungicides exerts selective pressure favoring fungicide-resistant strains of pathogens. Anecdotal reports and evidence from field experiments suggest that the efficacy of DMI fungicides to control CLS declined from the late 1980s when they were first introduced $(8,9,12)$ to $2002(13,16,18)$. In laboratory assays, some $B$. jaapii isolates from sites where DMI fungicides had been used intensively were not inhibited at DMI fungicide concentrations 100 times greater than the concentration that inhibited sensitive isolates (19).

Pyraclostrobin and trifloxystrobin, members of the QoI class of respiration inhibitor fungicides, were registered on sour cherry in late 2002. Pyraclostrobin is also packaged with boscalid, a respiration inhibitor with a mode of action different from that of the QoI fungicides, and the combination is marketed as Pristine (BASF Corp., Research Triangle Park, NC). Boscalid, pyraclostrobin, and trifloxystrobin have been deemed "reduced risk" by EPA, based on their low toxicity to mammals and wildlife. In field trials $(11,16,18)$, the QoI fungicides have proven effective against CLS, brown rot, and powdery mildew, all of which are common and economically important diseases in moist, temperate climates. Like the DMI fungicides, however, the QoI fungicides are prone to fungicide resistance (3). Therefore, it is critical to develop CLS management programs that exploit the strengths of the DMI and QoI fungicides but that are not entirely reliant on them.

The use of Bordeaux mixture (copper sulfate plus hydrated lime) for the control of CLS and other diseases of cherry dates to the late 1800 s (reviewed in 14). Following field trials in Wisconsin that spanned two decades, Keitt et al. (14) reported that although Bordeaux mixture was phytotoxic to sour cherry leaves and reduced fruit size slightly, its efficacy in controlling CLS far outweighed the negative side effects. Nev- 
ertheless, the use of Bordeaux on sour cherry diminished with the introduction of synthetic fungicides in the 1940s and 1950s. Because the mode of action of copper is nonspecific denaturation of proteins (24), fungi are not likely to become resistant to it. Furthermore, most copper-based fungicides are less expensive than DMIs, QoIs, or chlorothalonil, but are as convenient to use as synthetic fungicides. These features make copper an appealing option for CLS control. Nevertheless, most sour cherry growers are reluctant to incorporate copper-based fungicides into their spray programs without further data demonstrating their efficacy in controlling CLS.

Reliance on expensive, potentially carcinogenic fungicides to control CLS and the existence and continuing threat of fungicide resistance prompted us to evaluate spray programs that integrate copper-based and respiration inhibitor fungicides with the standards for CLS control, chlorothalonil and DMI fungicides. Another objective was to test the efficacy of spray programs in which chlorothalonil was eliminated. Some field experiments were conducted in parallel on the cultivars Montmorency and Balaton, because acreage of the latter is increasing, but the relative susceptibility of the two cultivars has not been formally evaluated. To assess the relative strengths and weaknesses of the fungicides at various stages in the infection process, the physical modes of action (23) of three fungicides toward $B$. jaapii were determined in a leaf-disk assay. Finally, isolates of $B$. jaapii that varied in sensitivity to DMI fungicides were tested in vitro for their sensitivity to copper. A preliminary report of our findings was published (17).

\section{MATERIALS AND METHODS}

Field trials. Experiments were conducted at the Michigan State University Northwest Horticultural Research Station near Traverse City (MI) and the University of Wisconsin Peninsular Agricultural Research Station (UW PARS) near Sturgeon Bay (WI) in 2003 through 2005. Treatments varied among the 10 field trials (Table 1), but a common objective among all trials was to integrate copper-based and respiration inhibitor fungicides with chlorothalonil and DMIs. In Table 1, treatment descriptions reflect the type of copper compound and number of sprays integrated into the program. "Standard" programs contained no copper-based fungicides. In "no chlorothalonil" programs, chlorothalonil was replaced by a different fungicide. A subset of experiments (MI and WI 2004; MI 2005) compared fungicide performance on two cultivars of sour cherry, Montmorency and Balaton. In Michigan, treatments applied to a 'Montmorency' block established in 1979 ('Montmorency' block A) were replicated five times on single-tree plots. B. jaapii isolates with reduced sensitivity to DMIs have been documented in this block of trees (19). Treatments applied to a mixed block of 'Montmorency' and 'Balaton' established in 1995 in Michigan were replicated four times on four-tree plots. In Wisconsin, treatments applied to a 'Montmorency' block established in 1995 and a 'Balaton' block established in 2000 were replicated five (2004 and 2005) or six (2003) times on single-tree plots. Although isolates of $B$. jaapii from the Wisconsin site have not been tested for resistance to DMI fungicides, field trial data suggest that sensitivity to DMIs has decreased at this site since the late 1980s (data reported in references 8 and 9 compared with 16 and 18). Fungicides were applied in 560 liters of water per hectare to the mixed 'Balaton' and 'Montmorency' block in Michigan using an airblast sprayer. In other blocks, fungicides were applied in 2,800 liters of water per hectare using handgun sprayers operated at 18 to 22 $\mathrm{kg} / \mathrm{cm}$.

Leaf spot infection periods based on leaf wetness duration and temperature (2) were monitored throughout the growing season each year with weather stations at the research stations in Michigan and Wisconsin. In most experiments, CLS incidence and defoliation were rated at about the time of harvest and again approximately 2 to 6 weeks after harvest (Table 2). In Michigan in 2003 and Wisconsin in 2005, a harvest rating was not conducted; in Michigan in 2005, a preharvest rating was made in late June (Table 2). Incidence was defined as the average percentage of leaves with CLS on 10 (WI) or 20 (MI) terminal shoots on each tree. Percent defoliation was defined as $1-$ (number of leaves/number of nodes) $\times 100$. Minor spotting of unknown etiology and damage from insects was sometimes noticed, but CLS was presumed to be the overwhelming cause of premature defoliation.

Statistical analysis of field data. The effects of treatments on CLS incidence and defoliation were analyzed separately for each trial. A square root transformation was applied to correct for unequal variances where necessary prior to analysis of variance. Means were separated according to Fisher's protected least significant difference test $(P=0.05)$. To determine whether 'Balaton' and 'Montmorency' varied in susceptibility to CLS, data collected on 24 August 2004 and 12 September 2005 from the mixed planting in Michigan were combined over treatments and analyzed separately for each year. Prior to analysis, an $\arctan (y+2)$ transformation was applied to correct for unequal variances. The effect of cultivar on CLS incidence and defoliation in each year was determined by one-way analysis of variance (GLM function of SAS, version 9.1.3, SAS Institute, Cary, NC), and means were separated with Fisher's protected least significant difference test $(P=0.05)$.
Fungicide physical modes of action. Protectant, postinfection, and antisporulant activities of three fungicides were assessed by a modification of the leaf disk inoculation method described by Wharton et al. (25). The B. jaapii inoculum used in these experiments originally was collected from UW PARS and was maintained on sour cherry leaf disks in vitro. Leaf disks were cut from fully expanded 'Montmorency' sour cherry leaves using an $18-\mathrm{mm}$ diameter cork borer (leaf disk area $=2.54$ $\mathrm{cm}^{2}$ ). Six disks were placed abaxial surface up on $1.5 \%$ water agar in four replicate petri plates for each treatment. Commercial formulations of trifloxystrobin (Flint 50WDG, $38 \mu \mathrm{g}$ a.i. $\mathrm{ml}^{-1}$ ), tebuconazole (Elite $45 \mathrm{DF}, 68 \mu \mathrm{g}$ a.i. $\mathrm{ml}^{-1}$ ), and basic copper sulfate (Cuprofix Disperss 20DF, $221 \mu \mathrm{g}$ copper ion $\mathrm{ml}^{-1}$ ) were dissolved in sterile water and applied to disks with an atomizer to evenly cover leaf tissue. Sterile water was applied as a control; a second control consisted of plates with disks that were not treated. Fungicides or water were applied either $0.5 \mathrm{~h}$ before inoculation (protectant) or $46 \mathrm{~h}$ after inoculation (postinfection) with $B$. jaapii $\left(4.6 \times 10^{5}\right.$ conidia $\mathrm{ml}^{-1}$ in trial $1 ; 5.5 \times 10^{5}$ conidia $\mathrm{ml}^{-1}$ in trial 2) and incubated at $20^{\circ} \mathrm{C}$ with a 12-h photoperiod provided by fluorescent lights. Fungicide antisporulant activity was assessed by applying fungicides or water to symptomatic leaf disks from which acervuli had been removed. For this, a separate set of leaf disks was inoculated and incubated, and after 10 days acervuli were removed by washing leaf disks for 15 min in sterile water until the conidial masses were no longer visible. Four washed disks were placed on water agar in each of four replicate petri plates per treatment. Following treatment with fungicides or water, plates were incubated at $20^{\circ} \mathrm{C}$ with a 12 -h photoperiod provided by fluorescent lights. The percentage of lesions on each disk with sporulating acervuli was recorded after 6 (trial 1) or 5 (trial 2) days. Data were square-root transformed prior to analysis of variance, and means were separated according to Fisher's protected least significant difference test $(P=0.05)$.

Sensitivity of $B$. jaapii to copper in vitro. In a previous study (19), isolates of $B$. jaapii were categorized based on their relative sensitivity to the DMI fungicide fenbuconazole. Fifty isolates representing four phenotypic resistance classes (sensitive, low moderate, high moderate, or resistant) were tested for their sensitivity to copper. The $B$. jaapii isolates were grown at 23 to $25^{\circ} \mathrm{C}$ on MMEA ( $2 \%$ malt extract, $0.1 \%$ yeast extract, $2 \%$ agar) (19) to produce conidia. Conidial suspensions were transferred to water agar ( $2 \%$ agar) containing $0,5,10,25$, or $50 \mu \mathrm{g} \mathrm{CuSO}_{4} \cdot 5 \mathrm{H}_{2} \mathrm{O}$ (EM Science, Gibbstown, NJ) per $\mathrm{ml}$. Plates were incubated at $23^{\circ} \mathrm{C}$ for 24 to 48 $\mathrm{h}$, after which percent germination was 
determined by evaluating 100 arbitrarily selected conidia on each of two replicate plates and combining the results. The experiment was performed twice.

\section{RESULTS}

Cherry leaf spot infection periods.

The dates and intensity of CLS infection periods (2) are reported in Table 3 . Discharge of B. jaapii ascospores, the primary inoculum for CLS, commences during bloom (10). Therefore, the earliest infection periods reported are those that occurred no more than 8 days prior to the first spray, which was applied at the bloom or shuck split stage (Table 1). In Michigan, the greatest number of infection periods occurred in 2005, but only four occurred in May and June. By contrast, in 2004, there were seven infection periods in May and June, three of which registered high intensity. In Wisconsin, the fewest infection periods occurred in 2005. By contrast, in 2004 seven infection periods occurred in

Table 1. Fungicide spray programs in Michigan and Wisconsin, 2003 through 2005

\begin{tabular}{|c|c|c|c|c|c|c|c|c|c|}
\hline \multirow[b]{2}{*}{$\begin{array}{l}\text { Year, location, } \\
\text { cultivar }\end{array}$} & \multirow[b]{2}{*}{$\begin{array}{l}\text { Treatment } \\
\text { description }^{x}\end{array}$} & \multicolumn{8}{|c|}{ Dates of phenological stages and fungicides $\left(\mathrm{g} \mathrm{ha}^{-1}\right)$ applied $^{\mathrm{w}}$} \\
\hline & & $\begin{array}{l}\text { Bloom or } \\
\text { petal fall }\end{array}$ & $\begin{array}{l}\text { Shuck } \\
\text { split }\end{array}$ & $\begin{array}{l}\text { 1st } \\
\text { Cover }\end{array}$ & $\begin{array}{l}\text { 2nd } \\
\text { Cover }\end{array}$ & $\begin{array}{l}\text { 3rd } \\
\text { Cover }\end{array}$ & $\begin{array}{l}\text { 4th } \\
\text { Cover }\end{array}$ & $\begin{array}{l}\text { 5th } \\
\text { Cover }\end{array}$ & $\begin{array}{l}\text { Post- } \\
\text { harvest }\end{array}$ \\
\hline \multirow{5}{*}{$\begin{array}{l}\text { 2003, MI } \\
\text { Montmorency } \\
\text { (block A) }\end{array}$} & & 20 May & 30 May & $10 \mathrm{Jun}$ & 19 Jun & 30 Jun & $14 \mathrm{Jul}$ &.$- y$ & -- \\
\hline & Control & -- & -- & -- & -- & -- & -- & -- & -- \\
\hline & Basic copper sulfate (1) & Ch 2,780 & Ch 2,780 & Te 189 & $\operatorname{Tr} 91$ & $\mathrm{Cu} 1,572$ & $\operatorname{Tr} 91$ & -- & -- \\
\hline & Basic copper sulfate (2) & Ch 2,780 & Ch 2,780 & $\mathrm{Cu} 1,572$ & Te 189 & $\operatorname{Tr} 91$ & Cu 1,572 & -- & -- \\
\hline & Standard & Ch 2,780 & Ch 2,780 & Te 189 & $\operatorname{Tr} 91$ & Te 189 & $\operatorname{Tr} 91$ & -- & -- \\
\hline \multirow{7}{*}{$\begin{array}{l}\text { 2003, WI } \\
\text { Montmorency }\end{array}$} & & 2 Jun & -- & 12 Jun & 20 Jun & $1 \mathrm{Jul}$ & $14 \mathrm{Jul}$ & -- & 12 Aug \\
\hline & Control & & -- & - & & -- & & -- & \\
\hline & Basic copper sulfate (1) & Ch 2,780 & -- & $\operatorname{Tr} 140$ & Te 189 & $\mathrm{Cu} 2,246$ & Te 189 & -- & Ch 2,780 \\
\hline & Basic copper sulfate (2) & Ch 2,780 & -- & $\operatorname{Tr} 140$ & $\mathrm{Cu} 2,246$ & $\mathrm{Cu} 2,246$ & Te 189 & -- & Ch 2,780 \\
\hline & Basic copper sulfate (3) & Ch 2,780 & -- & $\mathrm{Cu} 2,246$ & $\mathrm{Cu} 2,246$ & $\mathrm{Cu} 2,246$ & Te 189 & -- & Ch 2,780 \\
\hline & Standard A & Ch 2,780 & -- & P $94+$ B 185 & Te 189 & P $94+$ B 185 & Te 189 & -- & Ch 2,780 \\
\hline & Standard B & Ch 2,780 & -- & $\operatorname{Tr} 140$ & Te 189 & $\operatorname{Tr} 140$ & Te 189 & -- & Ch 2,780 \\
\hline \multirow{7}{*}{$\begin{array}{l}\text { 2004, MI } \\
\text { Montmorency } \\
\text { (block A) }\end{array}$} & & 17 May & 26 May & 4 Jun & 14 Jun & 24 Jun & 5 Jul & $15 \mathrm{Jul}$ & -- \\
\hline & Control & -- & -- & -- & -- & -- & -- & -- & -- \\
\hline & Basic copper sulfate (2) & Ch 2,780 & Ch 2,780 & $\operatorname{Tr} 105$ & $\mathrm{Cu} 1,572$ & $\mathrm{Cu} 1,572$ & Te 189 & $\operatorname{Tr} 105$ & -- \\
\hline & Basic copper sulfate (2) & Ch 2,780 & Ch 2,780 & $\mathrm{Cu} 1,572$ & $\operatorname{Tr} 105$ & $\mathrm{Cu} 1,572$ & Te 189 & $\operatorname{Tr} 105$ & -- \\
\hline & Basic copper sulfate (3) & Ch 2,780 & Ch 2,780 & $\mathrm{Cu} 1,572$ & $\mathrm{Cu} 1,572$ & $\mathrm{Cu} 1,572$ & Te 189 & $\operatorname{Tr} 105$ & -- \\
\hline & Standard A & Ch 2,780 & Ch 2,780 & P $94+\mathrm{B} 185$ & Te 189 & $\operatorname{Tr} 105$ & Te 189 & $\operatorname{Tr} 105$ & \\
\hline & Standard B & Ch 2,780 & Ch 2,780 & $\operatorname{Tr} 105$ & Te 189 & $\operatorname{Tr} 105$ & Te 189 & $\operatorname{Tr} 105$ & -- \\
\hline \multirow{8}{*}{$\begin{array}{l}\text { 2004, MI } \\
\text { Montmorency } \\
\text { and Balaton }\end{array}$} & & 18 May & 28 May & 7 Jun & 17 Jun & 29 Jun & 9 Jul & $19 \mathrm{Jul}$ & 9 Aug \\
\hline & Control & -- & -- & -- & -- & -- & -- & -- & -- \\
\hline & Basic copper sulfate (2) & Ch 2,780 & Ch 2,780 & $\operatorname{Tr} 140$ & $\mathrm{Cu} 2,246$ & $\mathrm{Cu} 2,246$ & Te 189 & $\operatorname{Tr} 105$ & Ch 2,780 \\
\hline & $\begin{array}{l}\text { Copper oxychloride/ } \\
\text { basic copper sulfate (2) }\end{array}$ & Ch 2,780 & Ch 2,780 & $\operatorname{Tr} 140$ & $\begin{array}{l}\text { Cu } 2,246 \\
\quad+\text { lime } 10,107\end{array}$ & $\begin{array}{l}\mathrm{Cu} 2,246 \\
\quad+\text { lime } 10,107\end{array}$ & Te 189 & Tr 105 & Ch 2,780 \\
\hline & Copper hydroxide (2) & Ch 2,780 & Ch 2,780 & $\operatorname{Tr} 140$ & $\mathrm{Cu} 2,246$ & Cu 2,246 & Te 189 & $\operatorname{Tr} 105$ & Ch 2,780 \\
\hline & Basic copper sulfate (3) & Ch 2,780 & Ch 2,780 & $\mathrm{Cu} 2,246$ & Cu 2,246 & $\mathrm{Cu} 2,246$ & Te 189 & $\operatorname{Tr} 105$ & Ch 2,780 \\
\hline & No chlorothalonil & Te 189 & Te 189 & $\operatorname{Tr} 189$ & $\mathrm{Cu} 1,572$ & $\operatorname{Tr} 140$ & Te 189 & $\operatorname{Tr} 105$ & $\mathrm{Cu} 1,572$ \\
\hline & Standard & Ch 2,780 & Ch 2,780 & $\operatorname{Tr} 140$ & Te 189 & $\operatorname{Tr} 140$ & Te 189 & $\operatorname{Tr} 105$ & Ch 2,780 \\
\hline \multirow{9}{*}{$\begin{array}{l}\text { 2004, WI } \\
\text { Montmorency } \\
\text { and Balaton }\end{array}$} & & $\ldots$ & 1 Jun & $10 \mathrm{Jun}$ & 18 Jun & 30 Jun & $10 \mathrm{Jul}$ & $27 \mathrm{Ju}$ & 16 or \\
\hline & Control & -- & 1 Jun & 10 Jun & 18 Jun & 30 Jun & $10 \mathrm{JuI}$ & $27 \mathrm{Jul}$ & 23 Aug \\
\hline & Basic copper sulfate (2) & -- & Ch 2,780 & $\operatorname{Tr} 140$ & $\mathrm{Cu} 2,246$ & $\mathrm{Cu} 2,246$ & Te 189 & Te 189 & Ch 2,780 \\
\hline & Copper hydroxide (2) & -- & Ch 2,780 & $\operatorname{Tr} 140$ & $\mathrm{Cu} 1,965$ & $\mathrm{Cu} 1,965$ & Te 189 & Te 189 & Ch 2,780 \\
\hline & Basic copper sulfate (3) & -- & Ch 2,780 & $\mathrm{Cu} 2,246$ & $\mathrm{Cu} 2,246$ & Cu 2,246 & Te 189 & Te 189 & Ch 2,780 \\
\hline & Copper hydroxide (3) & -- & Ch 2,780 & Cu 1,965 & Cu 1,965 & Cu 1,965 & Te 189 & Te 189 & Ch 2,780 \\
\hline & No chlorothalonil & -- & Te 189 & $\operatorname{Tr} 140$ & $\mathrm{Cu} 2,246$ & $\operatorname{Tr} 140$ & Te 189 & Te 189 & $\mathrm{Cu} 2,246$ \\
\hline & Standard $\mathrm{A}^{\mathrm{z}}$ & -- & Ch 2,780 & P $94+$ B 185 & Te 189 & P $94+$ B 185 & Te 189 & Te 189 & Ch 2,780 \\
\hline & Standard $\mathrm{B}^{\mathrm{z}}$ & -- & Ch 2,780 & $\operatorname{Tr} 140$ & Te 189 & $\operatorname{Tr} 140$ & Te 189 & Te 189 & Ch 2,780 \\
\hline \multirow{4}{*}{$\begin{array}{l}\text { Montmorency } \\
\text { and Balaton } \\
\text { 2005, MI }\end{array}$} & & 10 May & 24 May & 3 Jun & 13 Jun & 23 Jun & $4 \mathrm{Jul}$ & -- & -- \\
\hline & Control & -- & & & -- & & & -- & -- \\
\hline & Basic copper sulfate (2) & Ch 2,780 & Ch 2,780 & $\operatorname{Tr} 140$ & $\mathrm{Cu} 2,246$ & $\mathrm{Cu} 2,246$ & Te 189 & -- & -- \\
\hline & $\begin{array}{l}\text { Copper oxychloride/ } \\
\text { basic copper sulfate (2) }\end{array}$ & Ch 2,780 & Ch 2,780 & $\operatorname{Tr} 140$ & $\begin{array}{l}\mathrm{Cu} 2,246+ \\
\quad \text { lime } 10,107\end{array}$ & $\begin{array}{l}\mathrm{Cu} 2,246+ \\
\quad \text { lime } 10,107\end{array}$ & Te 189 & -- & -- \\
\hline \multirow{13}{*}{$\begin{array}{l}\text { 2005, WI } \\
\text { Balaton }\end{array}$} & Copper hydroxide (2) & Ch 2,780 & Ch 2,780 & $\operatorname{Tr} 140$ & $\mathrm{Cu} 2,246$ & $\mathrm{Cu} 2,246$ & Te 189 & -- & -- \\
\hline & Basic copper sulfate (3) & Ch 2,780 & Ch 2,780 & $\mathrm{Cu} 2,246$ & $\mathrm{Cu} 2,246$ & $\mathrm{Cu} 2,246$ & Te 189 & -- & -- \\
\hline & No chlorothalonil & Te 189 & Te 189 & $\operatorname{Tr} 140$ & $\mathrm{Cu} 2,246$ & $\operatorname{Tr} 140$ & Te 189 & -- & -- \\
\hline & Standard & Ch 2,780 & Ch 2,780 & $\operatorname{Tr} 140$ & Te 189 & $\operatorname{Tr} 140$ & Te 189 & -- & -- \\
\hline & & -- & 24 May & 3 Jun & 14 Jun & $2 \mathrm{Jul}$ & $19 \mathrm{Jul}$ & -- & 23 Aug \\
\hline & Control & -- & -- & -- & -- & -- & -- & -- & -- \\
\hline & Copper hydroxide (1) A & -- & Ch 2,780 & P $94+$ B 185 & $\mathrm{Cu} 1,965$ & P $94+$ B 185 & Te 189 & -- & Ch 2,780 \\
\hline & Copper hydroxide (1) B & -- & Ch 2,780 & Te 189 & Cu 1,965 & Te 189 & P $94+$ B 185 & -- & Ch 2,780 \\
\hline & Copper hydroxide (1) C & -- & Ch 2,780 & $\operatorname{Tr} 140$ & $\mathrm{Cu} 1,965$ & $\operatorname{Tr} 140$ & Te 189 & -- & Ch 2,780 \\
\hline & Copper hydroxide (2) A & -- & Ch 2,780 & $\mathrm{Cu} 1,965$ & P $94+$ B 185 & $\mathrm{Cu} 1,965$ & Te 189 & -- & Ch 2,780 \\
\hline & Copper hydroxide (2) B & -- & Ch 2,780 & Cu 1,965 & Te 189 & Cu 1,965 & Te 189 & -- & Ch 2,780 \\
\hline & Copper hydroxide (2) C & -- & Ch 2,780 & Cu 1,965 & Te 189 & $\mathrm{Cu} 1,965$ & P $94+$ B 185 & -- & Ch 2,780 \\
\hline & No chlorothalonil & -- & P $94+$ B 185 & Cu 1,965 & Te 189 & $\mathrm{Cu} 1,965$ & Te 189 & -- & P $94+$ B 185 \\
\hline
\end{tabular}

w Shuck split is the stage at which sepals abscise; cover sprays are fungicide applications made at approximately 2-week intervals. Fungicide common names, brand names, formulations used, and manufacturer: $\mathrm{Ch}=$ chlorothalonil (Bravo Ultrex 82.5WDG in MI or Bravo WeatherStik in WI, Syngenta Crop Protection, Inc., Greensboro, NC); $\mathrm{Cu}=$ copper hydroxide (Kocide 40DF in MI or Kocide 2000 in WI, Griffin L.L.C., Valdosta, GA); Cu = copper oxychloride/copper sulfate (COCS, Platte Chemical Co., Fremont, NB); $\mathrm{Cu}=$ basic copper sulfate (Cuprofix Disperss 20DF, Cerexagri, Inc., King of Prussia, PA); P = pyraclostrobin combined with B = boscalid (Pristine, BASF Corp., Research Triangle Park, NC); Te = tebuconazole (Elite 45DF, Bayer CropScience LP, Research Triangle Park, NC); and Tr $=$ trifloxystrobin (Flint 50WDG, Syngenta Crop Protection, Inc., Greensboro, NC). Amounts of copper refer to metallic copper equivalent applied. Numbers refer to amount of active ingredient applied $\left(\mathrm{g} \mathrm{ha}^{-1}\right)$.

$\mathrm{x}$ Treatment description refers to copper compound and number of times it was sprayed or to a program that lacks chlorothalonil. Capital letters are assigned to differentiate similarly described programs. No fungicides were applied in the "control" program. "Standard" programs included synthetic fungicides commonly used by growers.

y -- indicates that no fungicide was applied corresponding to that phenological stage.

z Two "standard" programs were applied to 'Montmorency' but not 'Balaton' in Wisconsin in 2004. 


\begin{tabular}{|c|c|c|c|c|c|}
\hline \multirow{3}{*}{$\begin{array}{l}\text { Year, location, } \\
\text { cultivar }\end{array}$} & \multirow[b]{3}{*}{ Treatment description ${ }^{y}$} & \multicolumn{4}{|c|}{ Cherry leaf spot rating ${ }^{x}$} \\
\hline & & \multicolumn{2}{|c|}{ Incidence $(\%)$} & \multicolumn{2}{|c|}{ Defoliation (\%) } \\
\hline & & Harvest & Postharvest & Harvest & Postharvest \\
\hline 2003, MI & & Not rated & 12 Sep & Not rated & 12 Sep \\
\hline \multirow[t]{4}{*}{ Montmorency } & Control & -- & $72 \mathrm{a}^{\mathrm{z}}$ & -- & $22 \mathrm{a}$ \\
\hline & Basic copper sulfate (1) & -- & $5 \mathrm{c}$ & -- & $2 \mathrm{~b}$ \\
\hline & Basic copper sulfate (2) & -- & $1 \mathrm{c}$ & -- & $1 \mathrm{~b}$ \\
\hline & Standard & -- & $14 \mathrm{~b}$ & -- & $2 \mathrm{~b}$ \\
\hline 2003, WI & & 1 Aug & 3 Sep & 1 Aug & 3 Sep \\
\hline \multirow{6}{*}{ Montmorency } & Control & $43 a$ & $96 \mathrm{a}$ & 2 & $87 \mathrm{a}$ \\
\hline & Basic copper sulfate (1) & $1 \mathrm{~b}$ & $28 \mathrm{c}$ & $<1$ & $4 \mathrm{~b}$ \\
\hline & Basic copper sulfate (2) & $1 \mathrm{~b}$ & $15 \mathrm{c}$ & 1 & $1 \mathrm{~b}$ \\
\hline & Basic copper sulfate (3) & $3 \mathrm{~b}$ & $19 \mathrm{c}$ & 2 & $1 \mathrm{~b}$ \\
\hline & Standard A & $<1 \mathrm{~b}$ & $20 \mathrm{c}$ & $<1$ & $2 \mathrm{~b}$ \\
\hline & Standard B & $0 \mathrm{~b}$ & $43 \mathrm{~b}$ & $<1$ & $9 \mathrm{~b}$ \\
\hline 2004, MI & & $29 \mathrm{Jul}$ & 26 Aug & 29 Jul & 26 Aug \\
\hline Montmorency & Control & $100 \mathrm{a}$ & $98 \mathrm{a}$ & $80 \mathrm{a}$ & $85 \mathrm{a}$ \\
\hline (block A) & Basic copper sulfate (2) A & $36 \mathrm{bc}$ & $26 \mathrm{~d}$ & $5 \mathrm{~b}$ & $4 \mathrm{c}$ \\
\hline & Basic copper sulfate (2) B & $26 \mathrm{~cd}$ & $20 \mathrm{~d}$ & $<1 \mathrm{~b}$ & $2 \mathrm{c}$ \\
\hline & Basic copper sulfate $(3)$ & $30 \mathrm{~cd}$ & $35 \mathrm{c}$ & $3 \mathrm{~b}$ & $11 \mathrm{~b}$ \\
\hline & Standard A & $28 \mathrm{~cd}$ & $23 \mathrm{~d}$ & $2 \mathrm{~b}$ & $4 \mathrm{c}$ \\
\hline & Standard B & $39 \mathrm{~b}$ & $49 \mathrm{~b}$ & $<1 \mathrm{~b}$ & $11 \mathrm{~b}$ \\
\hline 2004, MI & & $29 \mathrm{Jul}$ & 26 Aug & 29 Jul & 26 Aug \\
\hline Montmorency & Control & $98 \mathrm{a}$ & $95 \mathrm{a}$ & $39 a$ & $54 \mathrm{a}$ \\
\hline & Basic copper sulfate (2) & $5 \mathrm{c}$ & $12 \mathrm{c}$ & $1 \mathrm{~b}$ & $1 \mathrm{~b}$ \\
\hline & Copper oxychloride/basic copper sulfate (2) & $3 \mathrm{c}$ & $6 \mathrm{~cd}$ & $0 \mathrm{~b}$ & $<1 \mathrm{~b}$ \\
\hline & Copper hydroxide (2) & $7 \mathrm{c}$ & $15 \mathrm{c}$ & $3 \mathrm{~b}$ & $2 \mathrm{~b}$ \\
\hline & Basic copper sulfate (3) & $12 \mathrm{bc}$ & $25 \mathrm{~b}$ & $<1 \mathrm{~b}$ & $5 \mathrm{~b}$ \\
\hline & No chlorothalonil & $5 \mathrm{c}$ & $11 \mathrm{c}$ & $<1 \mathrm{~b}$ & $3 \mathrm{~b}$ \\
\hline & Standard & $3 c$ & $4 \mathrm{~d}$ & $0 \mathrm{~b}$ & $<1 \mathrm{~b}$ \\
\hline 2004, MI & & $29 \mathrm{Jul}$ & 26 Aug & 29 Jul & 26 Aug \\
\hline Balaton & Control & $99 \mathrm{a}$ & $99 \mathrm{a}$ & $24 \mathrm{a}$ & $41 \mathrm{a}$ \\
\hline & Basic copper sulfate $(2)$ & $7 \mathrm{bc}$ & $8 \mathrm{~b}$ & $<1 \mathrm{c}$ & $1 \mathrm{~b}$ \\
\hline & Copper oxychloride/basic copper sulfate (2) & $4 \mathrm{c}$ & $<1 \mathrm{c}$ & $6 \mathrm{bc}$ & $4 \mathrm{~b}$ \\
\hline & Copper hydroxide (2) & $5 \mathrm{c}$ & $<1 \mathrm{c}$ & $6 \mathrm{bc}$ & $2 \mathrm{~b}$ \\
\hline & Basic copper sulfate (3) & $7 \mathrm{bc}$ & $10 \mathrm{~b}$ & $0 \mathrm{c}$ & $2 \mathrm{~b}$ \\
\hline & No chlorothalonil & $12 \mathrm{~b}$ & $12 \mathrm{~b}$ & $<1 \mathrm{c}$ & $3 \mathrm{~b}$ \\
\hline & Standard & $4 \mathrm{c}$ & $4 \mathrm{c}$ & $<1 \mathrm{c}$ & $2 \mathrm{~b}$ \\
\hline 2004, WI & & 5 Aug & 19 Aug & 5 Aug & 19 Aug \\
\hline Montmorency & Control & $93 \mathrm{a}$ & Defoliated & $74 \mathrm{a}$ & $100 \mathrm{a}$ \\
\hline & Basic copper sulfate (2) & $22 \mathrm{~cd}$ & $32 \mathrm{bc}$ & $5 \mathrm{c}$ & $7 \mathrm{c}$ \\
\hline & Copper hydroxide (2) & $19 \mathrm{~cd}$ & $30 \mathrm{bc}$ & $4 \mathrm{c}$ & $3 \mathrm{c}$ \\
\hline & Basic copper sulfate (3) & $11 \mathrm{~d}$ & $19 \mathrm{c}$ & $4 \mathrm{c}$ & $9 \mathrm{c}$ \\
\hline & Copper hydroxide (3) & $22 \mathrm{~cd}$ & $35 \mathrm{bc}$ & $4 \mathrm{c}$ & $7 \mathrm{c}$ \\
\hline & No chlorothalonil & $48 \mathrm{~b}$ & $57 \mathrm{a}$ & $20 \mathrm{~b}$ & $22 \mathrm{~b}$ \\
\hline & Standard A & $30 \mathrm{c}$ & $35 \mathrm{bc}$ & $6 \mathrm{c}$ & $8 \mathrm{c}$ \\
\hline & Standard B & $30 \mathrm{c}$ & $39 \mathrm{~b}$ & $9 \mathrm{c}$ & $8 \mathrm{c}$ \\
\hline 2004, WI & & 5 Aug & 19 Aug & 5 Aug & 19 Aug \\
\hline Balaton & Control & $87 \mathrm{a}$ & Defoliated & $69 \mathrm{a}$ & $100 \mathrm{a}$ \\
\hline & Basic copper sulfate (2) & $43 \mathrm{c}$ & $48 \mathrm{bc}$ & $7 \mathrm{c}$ & $19 \mathrm{c}$ \\
\hline & Copper hydroxide (2) & $37 \mathrm{c}$ & $58 \mathrm{~b}$ & $9 \mathrm{a}$ & $31 \mathrm{c}$ \\
\hline & Copper sulfate (3) & $33 \mathrm{c}$ & $38 \mathrm{c}$ & $5 \mathrm{a}$ & $13 \mathrm{c}$ \\
\hline & Copper hydroxide (3) & $45 \mathrm{c}$ & $62 \mathrm{~b}$ & $7 \mathrm{a}$ & $28 \mathrm{c}$ \\
\hline & No chlorothalonil & $66 \mathrm{~b}$ & $84 \mathrm{a}$ & $26 \mathrm{~b}$ & $50 \mathrm{~b}$ \\
\hline 2005, MI & & Preharvest, 28 Jun & 12 Sep & Preharvest, 28 Jun & 12 Sep \\
\hline Montmorency & Control & $<1$ & $39 \mathrm{a}$ & $<1$ & 9 \\
\hline & Basic copper sulfate $(2)$ & $<1$ & $0 \mathrm{~b}$ & 1 & 5 \\
\hline & Copper oxychloride/basic copper sulfate (2) & 0 & $<1 \mathrm{~b}$ & 0 & 5 \\
\hline & Copper hydroxide (2) & 0 & $0 \mathrm{~b}$ & $<1$ & $<1$ \\
\hline & Copper sulfate (3) & 0 & $2 \mathrm{~b}$ & 0 & 8 \\
\hline & No chlorothalonil & 0 & $<1 \mathrm{~b}$ & $<1$ & 0 \\
\hline & Standard & 0 & $2 \mathrm{~b}$ & $<1$ & $<1$ \\
\hline MI, 2005 & & Preharvest, 29 Jun & 12 Sep & Preharvest, 29 Jun & 12 Sep \\
\hline Balaton & Control & 0 & $40 \mathrm{a}$ & $<1$ & 3 \\
\hline & Copper sulfate (2) & 0 & $0 \mathrm{~b}$ & 0 & $<1$ \\
\hline & Copper oxychloride/copper sulfate (2) & $<1$ & $<1 \mathrm{~b}$ & 0 & 2 \\
\hline & Copper hydroxide (2) & 0 & $<1 \mathrm{~b}$ & 0 & $<1$ \\
\hline & Copper sulfate (3) & 0 & $<1 \mathrm{~b}$ & 1 & 3 \\
\hline & No chlorothalonil & 0 & $0 \mathrm{~b}$ & $<1$ & 3 \\
\hline & Standard & 0 & $2 \mathrm{~b}$ & 1 & 2 \\
\hline 2005, WI & & Not rated & 7 Sep & Not rated & $7 \mathrm{Sep}$ \\
\hline Balaton & Control & -- & $67 \mathrm{a}$ & - & $32 \mathrm{a}$ \\
\hline & Copper hydroxide (1) A & -- & $30 \mathrm{~b}$ & -- & $3 \mathrm{~b}$ \\
\hline & Copper hydroxide (1) B & -- & $26 \mathrm{~b}$ & -- & $4 \mathrm{~b}$ \\
\hline & Copper hydroxide (1) C & -- & $38 \mathrm{~b}$ & -- & $5 \mathrm{~b}$ \\
\hline & Copper hydroxide (2) A & -- & $22 \mathrm{~b}$ & -- & $3 \mathrm{~b}$ \\
\hline & Copper hydroxide (2) B & -- & $31 \mathrm{~b}$ & -- & $2 b$ \\
\hline & Copper hydroxide (2) C & - & $15 \mathrm{~b}$ & -- & $4 \mathrm{~b}$ \\
\hline & Copper hydroxide (2), no chlorothalonil & -- & $20 \mathrm{~b}$ & -- & $4 \mathrm{~b}$ \\
\hline
\end{tabular}

${ }_{\mathrm{x}}$ In MI, treatments were replicated five times on single-tree plots or four times on four-tree plots. In WI, treatments were replicated five or six times on single-tree plots. Incidence was defined as percentage of leaves with CLS on 10 (WI) or 20 (MI) terminal shoots on each tree. Percent defoliation was defined as $1-$ (number of leaves/number of nodes) $\times 100$.

y Treatment description refers to copper compound and number of times sprayed or to a program that lacks chlorothalonil. Capital letters are assigned to differentiate similarly described programs. No fungicides were applied in the "control" program. "Standard" programs included synthetic fungicides commonly used by growers.

${ }^{\mathrm{z}}$ Within an experiment, values followed by the same letter are not significantly different according to Fisher's protected least significant difference test $(P=0.05)$. No letter indicates that differences among treatments were not significant $(P=0.05)$. 
were similarly sensitive to copper in vitro, suggesting that copper-based fungicides could be used to control DMI-resistant populations of $B$. jaapii. None of the 50 isolates tested produced conidia that could germinate at $50 \mathrm{ppm}$ copper sulfate; by comparison, the concentration of copper sulfate applied in the field would be 600 ppm if Cuprofix Disperss fungicide (36.9\% basic copper sulfate) were applied in 2,800 liters of water at the label rate of $4.53 \mathrm{~kg} \mathrm{ha}^{-1}$. In general, copper-based fungicides are less expensive than chlorothalonil, DMIs, and respiration inhibitor fungicides, which could result in significant cost savings. On a per hectare basis, one application of classic Bordeaux mixture costs $\$ 10$ to $\$ 20$, and fixed copper fungicides range from $\$ 18$ to $\$ 40$, whereas one application of a sterol inhibitor or QoI fungicide costs $\$ 67$ to $\$ 104$ (2006 prices).

Notwithstanding the benefits described above, there are valid concerns surrounding the use of copper-based fungicides on sour cherry. Most conspicuous in our studies was phytotoxicity to leaves in some trials. Holb and Schnabel (5) reported that copper hydroxide applied three times during bloom through petal fall reduced the size and caused necrosis of sour cherry spur-leaf clusters. These phytotoxic effects were worse in a wet year than in a dry year and in the cultivar Érdi bötermö than in Üjfehértói fürtös. In growth chamber studies, phytotoxicity to leaves was greater on sour cherry seedlings that were treated with copper and exposed to nightly dew than on seedlings on which dew did not form (4). In the present study, the leaf area

Table 5. Effect of fungicides on resporulation of Blumeriella jaapii from existing cherry leaf spot lesions $^{y}$

\begin{tabular}{lcc}
\hline \multirow{2}{*}{ Treatment } & \multicolumn{2}{c}{$\begin{array}{c}\text { Percentage of lesions } \\
\text { with acervuli }\end{array}$} \\
\cline { 2 - 3 } & Trial 1 & Trial 2 \\
\hline Trifloxystrobin & $79.3 \mathrm{~b}^{\mathrm{z}}$ & $51.8 \mathrm{ab}$ \\
Tebuconazole & $14.3 \mathrm{a}$ & $27.3 \mathrm{a}$ \\
Basic copper sulfate & $86.3 \mathrm{~b}$ & $75.3 \mathrm{bc}$ \\
Water & $96.0 \mathrm{~b}$ & $92.0 \mathrm{c}$ \\
LSD & 29.3 & 29.2 \\
$P$ & 0.002 & 0.001 \\
\hline
\end{tabular}

${ }^{y}$ Leaf disks (each $2.54 \mathrm{~cm}^{2}$ ) with lesions exuding acervuli of Blumeriella jaapii conidia were washed for $15 \mathrm{~min}$ in water until acervuli were no longer visible. Four washed disks were placed on water agar in a petri plate, and there were four replicate plates per treatment. Commercial formulations of trifloxystrobin (Flint 50WDG, $38 \mu \mathrm{g}$ a.i. $\mathrm{ml}^{-1}$ ), tebuconazole (Elite 45DF, $68 \mu \mathrm{g}$ a.i. $\mathrm{ml}^{-1}$ ), and basic copper sulfate (Cuprofix Disperss 20DF, $221 \mu \mathrm{g}$ copper ion $\mathrm{ml}^{-1}$ ) were applied to leaf disks with an atomizer. Values are the mean percentage of cherry leaf spot lesions with acervuli 6 days (trial 1) or 5 days (trial 2) after treatment with fungicides or water.

${ }^{z}$ Values within columns followed by the same letter are not significantly different according to Fisher's protected least significant difference test $(P=0.05)$. afflicted with phytotoxic lesions was not quantified, and therefore it cannot be correlated with the formulation of copper, number of applications, cultivar, leaf age, or environmental conditions. Leaf injury was generally minor, however, and had no obvious effect on yield or quality of fruit. Another concern is the effect of copper on benign and beneficial organisms in the orchard ecosystem. For example, excessive copper in soil is toxic to earthworms $(6,15)$. Likewise, copper is relatively toxic to certain fish and aquatic invertebrates, so copper-based fungicides should be used with great caution where runoff to surface water is likely. In 2005, EPA began reassessment of copper-based fungicides, which will draw scrutiny to the deleterious effects of copper and could sway future use patterns.

In addition to testing copper-based fungicides for CLS control, spray programs in which chlorothalonil was eliminated were tested. Substituting tebuconazole for chlorothalonil during the bloom through shuck-split stages decreased the level of CLS control in both Michigan and Wisconsin in 2004. However, in 2005, CLS control was not compromised by replacing chlorothalonil with tebuconazole in Michigan or with pyraclostrobin plus boscalid in Wisconsin. This may be attributed in part to the greater disease pressure in 2004, particularly in May and early June when primary infection from $B$. jaapii ascospores occurs. At both sites, there were more infection periods in May and June of 2004 than in the same months of 2005. Likewise, there were more high-intensity infection periods in 2004 than in 2005. Furthermore, the presence of DMIresistant strains of $B$. jaapii might have contributed to the failure of tebuconazole under high disease pressure in 2004. Better control of CLS might have been achieved in 2004 had a respiration inhibitor fungicide been substituted for chlorothalonil. Substituting a copper-based fungicide for chlorothalonil at the bloom through shucksplit stages might also be effective; however, as mentioned above, spur leaves are vulnerable to the toxic effects of copper applied during bloom (5).

Our leaf disk assays to test the physical modes of action of representative QoI (trifloxystrobin), DMI (tebuconazole), and copper-based (basic copper sulfate) fungicides suggest that QoI and copper-based fungicides will perform best when applied preventatively. Protectant activity of tebuconazole was erratic in these assays, and postinfection activity was disappointing considering that a key strength of DMI fungicides is inhibition of hyphal elongation. Despite its shortcomings in protectant and postinfection activity, tebuconazole was generally more effective than either trifloxystrobin or basic copper sulfate in preventing resporulation from existing lesions. Nevertheless, the use of tebucona- zole as an antisporulant is not recommended, as this practice would likely further the selection of B. jaapii strains with reduced sensitivity to DMI fungicides. Cuprofix Disperss (copper sulfate formulated with gypsum) did not inhibit resporulation from CLS lesions in the leaf disk assay and therefore does not show promise as an antisporulant.

Anecdotal reports from growers and researchers regarding the relative susceptibility of 'Balaton' and 'Montmorency' to CLS have been inconsistent. The mixed block of 'Balaton' and 'Montmorency' in Michigan was ideal to compare cultivars because trees were exposed to similar inoculum load and environmental conditions. Under high disease pressure in 2004 and relatively low disease pressure in 2005, there were no significant differences between the cultivars in CLS incidence or percent defoliation. Regression analysis was attempted to compare cultivars for the degree of defoliation at a given disease incidence. However, valid analyses were not possible because defoliation was consistently low (8\% or less) among treated trees, regardless of disease incidence. Defoliation was variable among nontreated control trees, but the number of data points was insufficient for a meaningful analysis. Although our data suggest that 'Balaton' and 'Montmorency' do not differ in susceptibility to CLS, their relative ability to retain leaves at a given disease incidence remains unknown.

In summary, our work has provided a basis for revisiting the use of copper-based fungicides in commercial production of sour cherry. Spray programs in which chlorothalonil was applied during the bloom through shuck-split stage followed by copper-based fungicides in early cover sprays were highly effective in controlling CLS. DMI and respiration inhibitor fungicides are effective against brown rot and powdery mildew $(11,16,18,22)$, and these fungicides were intentionally applied in fourth and fifth cover sprays to protect fruit from brown rot just prior to harvest. As a result of this study, integrated copper programs are being scaled up to include entire orchard blocks in research and commercial orchards in Michigan and Wisconsin. In these trials, the compatibility of copper-based fungicides with various insecticides is being monitored, and lime is being added to some treatments in an attempt to mitigate the phytotoxicity associated with copper-based fungicides. Additional studies are also underway to quantify the phytotoxicity and to assess the effect of copper on carbon assimilation, stomatal conductance, and nontarget microbes on cherry leaves.

\section{ACKNOWLEDGMENTS}

We thank M. Anderson, W. Klein, B. Schauske, M. Stasiak, and R. Weidman for assistance in the field, and N. Keuler for advice on statistics. This project was supported by the United States De- 
partment of Agriculture CSREES Risk Avoidance and Mitigation Program (2003-51101-02120), University of Wisconsin System Applied Research Grant Program, the Michigan Cherry Committee, and the Agricultural Experiment Stations of Michigan and Wisconsin. We also thank the Marco Polo Project, Alma UE, University of Bologna for supporting R. Berardi for a research internship at Michigan State University.

\section{LITERATURE CITED}

1. Downey, S. 1999. Genetic diversity of Prunus serotina and the evaluation of other wild species for breeding sour cherry resistant to cherry leaf spot. M.Sc. thesis. Michigan State University, East Lansing.

2. Eisensmith, S. P., and Jones, A. L. 1981. Infection model for timing of fungicide applications to control cherry leaf spot. Plant Dis. 65:955958.

3. Gisi, U., Chin, K. M., Knapova, G., Farber, R. K., Mohr, U., Parisi, S., Sierotzki, H., and Steinfeld, U. 2000. Recent developments in elucidating modes of resistance to phenylamide, DMI, and strobilurin fungicides. Crop Prot. 19:836-872.

4. Gruber, B. R., Kruger, E. L., and McManus, P. S. 2006. Effect of copper fungicides on tart cherry photosynthetic parameters. (Abstr.) Phytopathology 96:S43.

5. Holb, I. J., and Schnabel, G. 2005. Effect of fungicide treatments and sanitation practices on brown rot blossom blight incidence, phytoxicity, and yield for organic sour cherry production. Plant Dis. 89:1164-1170.

6. Holmstrup, M., Peterson, B., and Larsen, M. 1998. Combined effects of copper, desiccation, and frost on the viability of earthworm cocoons. Environ. Toxicol. Chem. 17:897-901.

7. Howell, G. S., and Stackhouse, S. S. 1973. The effect of defoliation time on acclimation and dehardening in tart cherry (Prunus cerasus L.). J. Am. Soc. Hortic. Sci. 98:132-136.

8. Jeffers, S. N., and Weidman, R. 1989. Evaluation of fungicides for management of sour cherry diseases in Wisconsin, 1988. Fungicide and Nematicide Tests 44:56. The American Phytopathological Society, St. Paul, MN.

9. Jeffers, S. N., and Weidman, R. 1990. Evaluation of fungicides for management of sour cherry diseases in Wisconsin, 1989. Fungicide and Nematicide Tests 45:40. The American Phytopathological Society, St. Paul, MN.

10. Jones, A. L. 1995. Cherry leaf spot. Pages 2122 in: Compendium of Stone Fruit Diseases. J. M. Ogawa, E. I. Zehr, G. W. Bird, D. F. Ritchie, K. Uriu, and J. K. Uyemoto, eds. American Phytopathological Society, St. Paul, $\mathrm{MN}$.

11. Jones, A. L., and Ehret, G. R. 2000. Evaluation of strobilurin fungicides for cherry leaf spot and brown rot control, 1999. Fungicide and Nematicide Tests 55:53. The American Phytopathological Society, St. Paul, MN.

12. Jones, A. L., Ehret, G. R., Garcia, S. M., Kesner, C. D., and Klein, W. M. 1993. Control of cherry leaf spot and powdery mildew on sour cherry with alternate-side applications of fenarimol, myclobutanil, and tebuconazole. Plant Dis. 77:703-706.

13. Jones, A. L., Ehret, G. R., Sundin, G. W., Nugent, J. E., and Klein, W. M. 2003. New fungicide evaluation on Montmorency tart cherries for leaf spot control. Fungicide and Nematicide Tests (online) Report 58:STF003. DOI: 10.1094/FN58. The American Phytopathological Society, St. Paul, MN.

14. Keitt, G. W., Blodgett, E. C., Wilson, E. E., and Magie, R. O. 1937. The epidemiology and control of cherry leaf spot. Univ. Wisc. Agric. Exp. Stn. Res. Bull. 132.

15. Ma, W. 1988. Toxicity of copper to lumbricid earthworms in sandy agricultural soils amended with $\mathrm{Cu}$-enriched organic waste materials. Ecol. Bull. 39:53-56.

16. McManus, P. S., Stasiak, M., Schauske, B., and Weidman, R. 2003. Evaluation of fungicides for control of sour cherry diseases in Wisconsin, 2002. Fungicide and Nematicide Tests (online) Report 58:STF017. DOI: 10.1094/FN58. The American Phytopathological Society, St. Paul, MN.
17. McManus, P., Sundin, G., Proffer, T., Berardi, R., Ma, Z., and Ehret, G. 2005. Integration of copper, strobilurin, and sterol demethylation inhibitor fungicides for control of cherry leaf spot. (Abstr.) Phytopathology 95:S68.

18. McManus, P., and Weidman, R. 2001. Evaluation of fungicides for control of sour cherry diseases in Wisconsin, 2000. Fungicide and Nematicide Tests (online) Report 56:STF1. DOI: 10.1094/FN56. The American Phytopathological Society, St. Paul, MN.

19. Proffer, T. J., Berardi, R., Ma, Z., Nugent, J. E., Ehret, G. R., McManus, P. S., Jones, A. L. and Sundin, G. W. 2006. Occurrence, distribution, and PCR-based detection of resistance to sterol demethylation-inhibiting fungicides in populations of Blumeriella jaapii in Michigan. Phytopathology 96:709-717.

20. Sisler, H. D. 1994. Fungicidal action and fungal resistance mechanisms. Pages 6-8 in Fungicide Resistance in North America. C. J. Delp, ed. American Phytopathological Society, St. Paul, MN

21. Sjulin, T. M., Jones, A. L., and Andersen, R. L. 1989. Expression of partial resistance to cherry leaf spot in cultivars of sweet, sour, duke, and European ground cherry. Plant Dis. 73:56-61.

22. Sundin, G. W., Ehret, G. R., McManus, P. S. Nugent, J. E., Klein, W. M., Anderson, M. D., and Proffer, T. J. 2005. Evaluation of new fungicide rotations for cherry leaf spot control on Montmorency tart cherries in NW Michigan, 2004. Fungicide and Nematicide Tests (online) Report 60:STF004. DOI: 10.1094/FN60. The American Phytopathological Society, St. Paul, $\mathrm{MN}$.

23. Szkolnik, M. 1981. Physical modes of action of sterol-inhibiting fungicides against apple diseases. Plant Dis. 65:981-985.

24. Ware, G. W., and Whitacre, D. M. 2004. Fungicides and bactericides. Pages 155-172 in The Pesticide Book, 6th ed. MeisterPro Information Resources, Willoughby, $\mathrm{OH}$.

25. Wharton, P., Iezzoni, A., and Jones, A. 2003. Screening cherry germ plasm for resistance to leaf spot. Plant Dis. 87:471-477. 